

\title{
Geometry of quiver Grassmannians of Kronecker type and applications to cluster algebras
}

\author{
Giovanni Cerulli Irelli and Francesco Esposito
}

\begin{abstract}
We study quiver Grassmannians associated with indecomposable representations (of finite dimension) of the Kronecker quiver. We find a cellular decomposition for them and we compute their Betti numbers. As an application, we find a geometric realization for the atomic basis of cluster algebras of type $A_{1}^{(1)}$ found by Sherman and Zelevinsky (who called it the canonical basis) and those of type $A_{2}^{(1)}$ found in an earlier paper of the first author.
\end{abstract}

\section{Introduction}

Cluster algebras are commutative $\mathbb{Z}$-subalgebras of the field of rational functions in a finite number of indeterminates which have been introduced and studied in [Fomin and Zelevinsky 2002; 2003a; 2007; Berenstein et al. 2005]. To every quiver $Q$ without loops and 2-cycles it is associated a coefficient-free cluster algebra $\mathscr{A}_{Q}$. In [Caldero and Chapoton 2006; Caldero and Keller 2006; 2008; Derksen et al. 2010] the authors describe the cluster variables of $\mathscr{A}_{Q}$ via a map, called the CalderoChapoton map, between the representations of $Q$ and the field of rational functions in $n$ variables (we address the reader to the survey [Keller 2010]). This map is defined in terms of the Euler-Poincaré characteristic of some complex projective varieties attached to every finite-dimensional representation $M$ of $Q$ and called quiver Grassmannians. By definition, the quiver $\operatorname{Grassmannian} \mathrm{Gr}_{\boldsymbol{e}}(M)$ consists of all subrepresentations of $M$ of dimension vector $\boldsymbol{e}$. These varieties have been considered in, for example, [Caldero and Keller 2008; Caldero and Keller 2006; Caldero and Reineke 2008; Hernandez and Leclerc 2010, §12.3; Nakajima 2011]. In this paper we obtain more geometric information about them in the case of the Kronecker quiver. In [Caldero and Zelevinsky 2006] the authors compute the EulerPoincare characteristic of quiver Grassmannians associated with the Kronecker

Research supported by grant CPDA071244/07 of Padova University.

MSC2000: primary 06B15; secondary 16G20, 14N05, 13F99, 16G99, 05E10.

Keywords: complex algebraic geometry, quiver Grassmannians, cluster algebras, quiver representations. 
quiver and they conjecture the existence of a cellular decomposition which we find here. In [Cerulli Irelli 2011b] (see also [Poettering 2010]) a torus action on some quiver Grassmannians has been found and this allows to produce a cellular decomposition of them in the case they are smooth.

In order to get more concrete results, such as an explicit computation of Poincaré polynomials, we restrict our attention to the case of the Kronecker quiver. So in this paper we study quiver Grassmannians associated with finite-dimensional representations of the Kronecker quiver. A finite-dimensional representation of the Kronecker quiver (called from now on a $Q$-representation) is a quadruple $M=\left(M_{1}, M_{2}, m_{a}, m_{b}\right)$, where $M_{1}$ and $M_{2}$ are finite-dimensional complex vector spaces and $m_{a}, m_{b}: M_{1} \rightarrow M_{2}$ are two linear maps between them. Given two nonnegative integers $e_{1}$ and $e_{2}$, the variety $\operatorname{Gr}_{\left(e_{1}, e_{2}\right)}(M)$ is defined as the set

$$
\left\{\left(N_{1}, N_{2}\right) \in \mathrm{Gr}_{e_{1}}\left(M_{1}\right) \times \mathrm{Gr}_{e_{2}}\left(M_{2}\right): m_{a}\left(N_{1}\right) \subset N_{2}, m_{b}\left(N_{1}\right) \subset N_{2}\right\},
$$

where $\mathrm{Gr}_{e}(V)$ denotes the Grassmannian of $e$-dimensional vector spaces in a vector space $V$. This is a projective variety which is in general not smooth. When $M_{1}=$ $M_{2}=\mathbb{C}^{n}$ and $m_{a}=\mathrm{Id}$ is the identity matrix and $m_{b}=J_{n}(0)$ is an indecomposable nilpotent Jordan block, the representation $M$ is regular indecomposable and we denote it by $R_{n}$. The corresponding quiver Grassmannians $X=\operatorname{Gr}_{\left(e_{1}, e_{2}\right)}\left(R_{n}\right)$ are the main subjects of this paper. We concentrate on $R_{n}$ because it is an indecomposable (finite-dimensional) nonrigid $Q$-representation; all the other indecomposable (finitedimensional) $Q$-representations have either the same quiver Grassmannians as $R_{n}$ or they are rigid and hence their quiver Grassmannians are smooth and our results are already known for them (see Section 2.1). In Section 2.2 we find that a onedimensional torus $T$ acts on $X$. We provide a stratification of $X$ (see Section 2.5)

$$
X=X_{0} \supseteq X_{1} \supseteq \cdots \supseteq X_{s}
$$

for $s=\min \left(e_{1}, n-e_{2}\right)$ into closed subvarieties $X_{k} \simeq \operatorname{Gr}_{\left(e_{1}-k, e_{2}-k\right)}\left(R_{n-2 k}\right)$. Moreover $X_{k+1}$ is the singular locus of $X_{k}$ and the difference $X_{k} \backslash X_{k+1}$ is a smooth quasiprojective variety which is not complete. As a consequence of the stratification (1) we get that $X$ is smooth if and only if $s=0$, i.e., either $e_{1}=0$ or $e_{2}=n$, in which cases the quiver Grassmannian specializes to an usual Grassmannian of vector subspaces.

In Section 2.6 we prove that Białynicki-Birula's theorem on cellular decomposition of smooth projective varieties applies to $X_{k} \backslash X_{k+1}$; hence we can prove that $X$ has a decomposition

into attracting sets

$$
X=\bigcup_{L \in X^{T}} X_{L}
$$

$$
X_{L}:=\left\{N \in X: \lim _{\lambda \rightarrow 0} t_{\lambda} N=L\right\}
$$


of $T$-fixed points $L$, and theses sets are affine spaces. In Section 2.7 we describe the cell $X_{L}$ : if $L$ is indecomposable then $X_{L}$ is the orbit of $L$ by the action of a unipotent group; if $L=L^{\prime} \oplus L^{\prime \prime}$, with $L^{\prime}$ of "lower weight" than $L^{\prime \prime}$, we have

$$
\operatorname{dim} X_{L^{\prime} \oplus L^{\prime \prime}}=\operatorname{dim} X_{L^{\prime}}+\operatorname{dim} X_{L^{\prime}}-\left\langle\operatorname{dim} L^{\prime}, \operatorname{dim} L^{\prime \prime}\right\rangle,
$$

where $\langle\cdot, \cdot\rangle$ denotes the Euler form associated with the Kronecker quiver. As a consequence of this formula we are able to compute the Poincaré polynomials of the quiver Grassmannians associated with every finite-dimensional indecomposable representation of the Kronecker quiver: for $n \geq 0$ let $P_{n}$ (resp. $I_{n}$ ) be the indecomposable preprojective of dimension vector $(n, n+1)($ resp. $(n+1, n))$. As usual we denote by $P_{X}(t):=\sum_{i} \operatorname{dim} H^{i}(X) t^{i}$ the Poincaré polynomial of the complex projective variety $X$. Denoting by $\operatorname{Gr}_{t}(s)$ the Grassmannian of $t$-dimensional vector subspaces of an $s$-dimensional vector space, we have (see Section 2.8)

$$
\begin{aligned}
P_{\mathrm{Gr}_{e}\left(R_{n}\right)}(t) & =P_{\mathrm{Gr}_{\left(e_{2}-e_{1}\right)}\left(e_{2}\right)}(t) P_{\mathrm{Gr}_{\left(e_{2}-e_{1}\right)}\left(n-e_{1}\right)}(t), \\
P_{\mathrm{Gr}_{e}\left(P_{n}\right)}(t) & =P_{\mathrm{Gr}_{e_{1}}\left(e_{2}-1\right)}(t) P_{\mathrm{Gr}_{\left(e_{2}-e_{1}\right)}\left(n+1-e_{1}\right)}(t), \\
P_{\mathrm{Gr}_{e}\left(I_{n}\right)}(t) & =P_{\mathrm{Gr}_{e_{1}}\left(e_{2}+1\right)}(t) P_{\mathrm{Gr}_{\left(e_{2}-e_{1}\right)}\left(n-e_{1}\right)}(t),
\end{aligned}
$$

where $\boldsymbol{e}:=\left(e_{1}, e_{2}\right)$ denotes a dimension vector.

Szanto [2011] computed the polynomials which count the number of $\mathbb{F}_{q}$-points of the quiver Grassmannians associated with indecomposable (finite-dimensional) $Q$-representations. The polynomials he finds are precisely the ones we find: the Poincaré polynomials. This result is expected and should follow by standard technique in $\ell$-adic cohomology of schemes. It would deserve to be analyzed in more detail. In particular it would be interesting to know if the cellular decomposition continues to hold in positive characteristic.

In Section 3 we consider the (coefficient-free) cluster algebra $\mathscr{A}$ of type $A_{1}^{(1)}$. The cluster variables of $\mathscr{A}$ are the rational functions $x_{m}, m \in \mathbb{Z}$, of the field $\mathscr{F}=\mathbb{Q}\left(x_{1}, x_{2}\right)$ recursively generated by the relation

$$
x_{k} x_{k+2}=x_{k+1}^{2}+1
$$

It is not hard to see that every pair $\left\{x_{k}, x_{k+1}\right\}$ of consecutive cluster variables is a free-generating system for the field $\mathscr{F}$ and hence $\mathscr{F}_{F}=\mathbb{Q}\left(x_{k}, x_{k+1}\right)$ and every cluster variable can be expressed as a rational function in every such pair (which are called the clusters of $\mathscr{A}$ ). Every cluster variable is a Laurent polynomial in every cluster of $A$; this is the Laurent phenomenon proved in [Fomin and Zelevinsky 2002]. In this case the Caldero-Chapoton map $M \mapsto C C(M)$ associates to a $Q$-representation $M$ of dimension vector $\left(d_{1}, d_{2}\right)$ the Laurent polynomial 


$$
C C(M):=\frac{\sum_{\boldsymbol{e}} \chi\left(\mathrm{Gr}_{\boldsymbol{e}}(M)\right) x_{1}^{2\left(d_{2}-e_{2}\right)} x_{2}^{2 e_{1}}}{x_{1}^{d_{1}} x_{2}^{d_{2}}}
$$

Caldero and Keller [2008] proved that the map $M \mapsto C C(M)$ restricts to a bijection between the indecomposable rigid $Q$-representations $M$ (i.e., $\operatorname{Ext}^{1}(M, M)=0$ ) and the cluster variables of $\mathscr{A}$ different from $x_{1}$ and $x_{2}$. Moreover it has the property that $C C(M \oplus N)=C C(M) C C(N)$, under which cluster monomials not divisible by $x_{1}$ or $x_{2}$, i.e., monomials of the form $x_{k}^{a} x_{k+1}^{b}$ for $k \in \mathbb{Z} \backslash\{1,2\}$ and $a, b \geq 0$, are in bijection with rigid $Q$-representations.

Sherman and Zelevinsky [2004] introduced distinguished elements $\left\{z_{n}: n \geq 0\right\}$ of $\mathscr{F}$ recursively defined by

$$
z_{0}=2, \quad z_{1}=x_{0} x_{3}-x_{1} x_{2}, \quad z_{n+1}=z_{1} z_{n}-z_{n-1} \quad \text { for } n \geq 1,
$$

and proved that the set

$$
\boldsymbol{B}:=\{\text { cluster monomials }\} \cup\left\{z_{n}: n \geq 1\right\}
$$

is a $\mathbb{Z}$-basis of $\mathscr{A}$ such that positive linear combinations of its elements coincide with the set of all positive elements of $\mathscr{A}$, that is, elements that are positive Laurent polynomials in every cluster of $\mathscr{A}$. Such a basis is now called an atomic basis of $\mathscr{A}$, though Sherman and Zelevinsky called it a canonical basis. The terminology changed after the relationship between this basis and Lusztig's canonical basis was better understood; see [Lampe 2011; Geiß et al. 2012]. We give a new geometric realization of $\boldsymbol{B}$ by using the Caldero-Chapoton map: cluster monomials are images of rigid representations and quiver Grassmannians associated with rigid quiver representations are smooth [Caldero and Reineke 2008]. With this in mind we prove in Section 3 that

$$
z_{n}=\frac{\sum_{\boldsymbol{e}} \chi\left(\mathrm{Gr}_{\boldsymbol{e}}\left(R_{n}\right)^{\mathrm{sm}}\right) x_{1}^{2\left(n-e_{2}\right)} x_{2}^{2 e_{1}}}{x_{1}^{n} x_{2}^{n}}
$$

for every $n \geq 1$, where $\operatorname{Gr}_{e}\left(R_{n}\right)^{\mathrm{sm}}:=X_{0} \backslash X_{1}$ denotes the smooth part of $\operatorname{Gr}_{e}\left(R_{n}\right)$.

A similar construction can be made in a cluster algebra $\mathscr{A}_{2}$ of type $A_{2}^{(1)}$. These cluster algebras are studied in [Cerulli Irelli 2009] and some results are recalled in Section 3.2: the atomic basis of $\mathscr{A}_{2}$ consists of cluster monomials together with elements $\left\{u_{n} w^{k}, u_{n} z^{k}: k \geq 0, n \geq 1\right\}$ where $w$ and $z$ are two cluster variables and the $u_{n}$ are defined similarly to (4) as follows:

$$
u_{0}=2, \quad u_{1}=z w-2, \quad u_{n+1}=u_{1} u_{n}-u_{n-1} \quad \text { for } n \geq 1 .
$$

We prove that $u_{n}$ is obtained by evaluating the Caldero-Chapoton map at the smooth part of the regular indecomposable representation of a quiver of type $A_{2}^{(1)}$ of dimension vector $(n, n, n)$ that lies in a homogeneous tube. 


\section{Geometric structure of quiver Grassmannians}

Let

$$
Q:=1 \stackrel{a}{\stackrel{b}{\longrightarrow}} 2
$$

be the Kronecker quiver. As usual, we denote a complex $Q$-representation $M=$ $\left(M_{1}, M_{2}, m_{a}, m_{b}\right)$ as follows:

$$
M=M_{1} \stackrel{m_{a}}{\stackrel{m_{b}}{\longrightarrow}} M_{2} .
$$

A subrepresentation $N$ of $M$ consists of vector subspaces $N_{1}$ and $N_{2}$ of $M_{1}$ and $M_{2}$ respectively such that $m_{a}\left(N_{1}\right) \subset N_{2}$ and $m_{b}\left(N_{1}\right) \subset N_{2}$. We call

$$
\operatorname{dim}(M):=\left(\operatorname{dim} M_{1}, \operatorname{dim} M_{2}\right)
$$

the dimension vector of $M$. A morphism $g: M \rightarrow M^{\prime}$ from a $Q$-representation $M$ to a $Q$-representation $M^{\prime}$ is a pair $\left(g_{1}, g_{2}\right)$ of linear maps $g_{1}: M_{1} \rightarrow M_{1}^{\prime}$ and $g_{2}: M_{2} \rightarrow M_{2}^{\prime}$ such that $m_{a}^{\prime} \circ g_{1}=g_{2} \circ m_{a}$ and $m_{b}^{\prime} \circ g_{1}=g_{2} \circ m_{b}$. The set of $Q$-representations form a category which is an abelian Krull-Schmidt category via the natural notions of direct sums, kernel and cokernel (see [Assem et al. 2006], for example). The classification of finite-dimensional $Q$-representations which are indecomposable - that is, not direct sums of nontrivial subrepresentations - goes back to [Kronecker 1890]. Here is their complete list.

There are the indecomposable preprojectives

$$
P_{n}=k^{n} \underset{\varphi_{2}}{\stackrel{\varphi_{1}}{\longrightarrow}} k^{n+1},
$$

for $n \geq 0$, where $k=\mathbb{C}$ denotes the field of complex numbers, $k^{n}$ and $k^{n+1}$ denote complex vector spaces endowed respectively with linear bases $\left\{v_{1}^{(1)}, \ldots, v_{n}^{(1)}\right\}$ and $\left\{v_{1}^{(2)}, \ldots, v_{n+1}^{(2)}\right\}$, and $\varphi_{1}, \varphi_{2}$ are the immersions in the vector subspace of $k^{n+1}$ spanned by $\left\{v_{1}^{(2)}, \ldots, v_{n}^{(2)}\right\}$ and $\left\{v_{2}^{(2)}, \ldots, v_{n+1}^{(2)}\right\}$, respectively.

There are the indecomposable regulars

$$
R_{n}(\lambda)=k^{n} \underset{J_{n}(\lambda)}{\stackrel{\mathrm{Id}}{\longrightarrow}} k^{n}, \quad R_{n}(\infty)=k^{n} \underset{\mathrm{Id}}{\stackrel{J_{n}(0)}{\longrightarrow}} k^{n},
$$

where $\lambda \in k, J_{n}(\lambda)$ denotes the $n$-th indecomposable Jordan block of eigenvalue $\lambda$ and Id is the identity matrix (in the introduction we used the notation $R_{n}:=R_{n}(0)$ ).

Finally, there are the indecomposable preinjectives

$$
I_{n}=k^{n+1} \underset{\varphi_{1}^{t}}{\stackrel{\varphi_{2}^{t}}{\longrightarrow}} k^{n}
$$

for $n \geq 0$, where $\varphi_{1}^{t}$ and $\varphi_{2}^{t}$ are transposes of the matrices $\varphi_{1}$ and $\varphi_{2}$ defined above. 
For an indecomposable $Q$-representation $M$ we denote by $\mathscr{B}_{i}=\left\{v_{k}^{(i)}\right\}$, where $i=1,2$, the basis of $M_{i}$ with respect to which $M$ has the previous presentation. All other finite-dimensional $Q$-representations are direct sums of these ones. Direct sums of indecomposable preprojectives (resp. regulars, preinjectives) are called preprojective (resp. regular, preinjective) $Q$-representations.

Given nonnegative integers $e_{1}$ and $e_{2}$ and a $Q$-representation $M$ we consider the variety

$$
\operatorname{Gr}_{e}(M):=\left\{N \leq Q M: \operatorname{dim}(N)=\left(e_{1}, e_{2}\right)\right\},
$$

called the $\boldsymbol{e}=\left(e_{1}, e_{2}\right)$-quiver Grassmannian of $M$ (here $N \leq{ }_{Q} M$ means that $N$ is a subrepresentation of $M)$. This is closed inside the product $\mathrm{Gr}_{e_{1}}\left(M_{1}\right) \times \mathrm{Gr}_{e_{2}}\left(M_{2}\right)$ of usual Grassmannians of vector subspaces and so is a complex projective variety. In [Caldero and Reineke 2008] it is shown that the tangent space $T_{N}\left(\mathrm{Gr}_{e}(M)\right)$ at a point $N$ of $\operatorname{Gr}_{e}(M)$ equals:

$$
T_{N}\left(\operatorname{Gr}_{\boldsymbol{e}}(M)\right)=\operatorname{Hom}(N, M / N) .
$$

The following inequalities hold for $Z:=\mathrm{Gr}_{e}(M)$ :

$$
\langle\boldsymbol{e}, \boldsymbol{d}-\boldsymbol{e}\rangle \leq \operatorname{dim} Z \leq \operatorname{dim} T_{N}(Z) \leq\langle\boldsymbol{e}, \boldsymbol{d}-\boldsymbol{e}\rangle+\operatorname{dim} \operatorname{Ext}^{1}(M, M),
$$

where $\left\langle(a, b)^{t},(c, d)^{t}\right\rangle:=a c+b d-2 a d$ is the Euler form of $Q$ and $\boldsymbol{d}:=\operatorname{dim} M$, so that $\operatorname{dim}(M / N)=\boldsymbol{d}-\boldsymbol{e}$. In particular, if $M$ is $\operatorname{rigid}\left(\operatorname{Ext}^{1}(M, M)=0\right)$, then all the quiver Grassmannians associated with it are smooth (see [Derksen et al. 2010, Proposition 3.5] for a generalization of this result) and they have dimension $\operatorname{dim} \operatorname{Gr}_{\boldsymbol{e}}(M)=\langle\boldsymbol{e}, \boldsymbol{d}-\boldsymbol{e}\rangle$. It is known that the rigid $Q$-representations are

$$
P_{n}^{\oplus a} \oplus P_{n+1}^{\oplus b}, \quad I_{n}^{\oplus a} \oplus I_{n+1}^{\oplus b}
$$

for all $n \geq 0$ and $a, b \geq 0$.

2.1. Action of a group on quiver Grassmannians. Let $M$ be a $Q$-representation of dimension vector $\boldsymbol{d}=\left(d_{1}, d_{2}\right)$. We consider the group

$\mathcal{N}(M):=\left\{(A, B) \in \prod_{i=1}^{2} \mathrm{GL}_{d_{i}}\left(M_{i}\right): m_{a} A=B m_{a}, m_{b} A=\lambda B m_{b}\right.$ for some $\left.\lambda \in \mathbb{C}^{*}\right\}$.

Note that the automorphism group of $M$ is a closed subgroup of $\mathcal{N}(M)$ (for $\lambda=1)$. The group $\mathcal{N}(M)$ acts on $\operatorname{Gr}_{\boldsymbol{e}}(M)$ as follows $(A, B) \cdot\left(N_{1}, N_{2}\right):=\left(A N_{1}, B N_{2}\right)$.

Lemma 1. (1) For every $n \geq 0, \mathcal{N}\left(P_{n}\right) \simeq \mathbb{C}^{*} \times \mathbb{C}^{*}$.

(2) For every $n \geq 0, \mathcal{N}\left(I_{n}\right) \simeq \mathbb{C}^{*} \times \mathbb{C}^{*}$.

Proof. It follows easily from the definition that $\mathcal{N}\left(P_{n}\right)$ consists of diagonal matrices $(A, B)$ of the form

$$
A=\operatorname{diag}\left(a, a \lambda, \ldots, a \lambda^{n-1}\right), \quad B=\operatorname{diag}\left(a, a \lambda, \ldots, a \lambda^{n}\right)
$$


for $a, \lambda \in \mathbb{C}^{*}$. Similarly for $\mathcal{N}\left(I_{n}\right)$.

Proposition 2. Let $M$ be a rigid $Q$-representation. Then every quiver Grassmannian $\mathrm{Gr}_{\boldsymbol{e}}(M)$ associated with $M$ has a cellular decomposition.

Proof. The variety $\operatorname{Gr}_{e}(M)$ is smooth and $\mathcal{N}(M) \supset \mathbb{C}^{*}=: T$. The torus $T$ acts with finitely many fixed points. It follows hence by Białynicki-Birula results [BiałynickiBirula 1973] (see also [Chriss and Ginzburg 1997, §2.4]) that it has a cellular decomposition into attracting sets of its $T$-fixed points.

In the rest of the paper we mainly concentrate on the quiver Grassmannians associated with indecomposable regular $Q$-representations.

2.2. The variety $X=\mathbf{G r}_{e}\left(\boldsymbol{R}_{n}\right)$. From now on we will focus on quiver Grassmannians associated with indecomposable regular $Q$-representations. It is not hard to show that $\operatorname{Gr}_{e}\left(R_{n}(\lambda)\right)=\mathrm{Gr}_{e}\left(R_{n}(\mu)\right)$ for every $\lambda, \mu \in k \cup\{\infty\}$ (see [Cerulli Irelli 2011b], for example) and hence we consider the variety $X:=\operatorname{Gr}_{e}\left(R_{n}\right)$ (recall that our convention is $\left.R_{n}:=R_{n}(0)\right)$. It follows from the definition that

$$
X=\left\{N_{1} \subset N_{2} \subset k^{n}: J_{n}(0) N_{1} \subset N_{2}, \operatorname{dim} N_{i}=e_{i}, i=1,2\right\}
$$

and hence $X$ is a closed subvariety of a partial flag variety. In this paper we use the convention that

$$
J=J_{n}(0) \text { is a lower triangular matrix. }
$$

The group $\mathcal{N}:=\mathcal{N}\left(R_{n}\right)$ is given by

$$
\mathcal{N}:=\left\{A \in \mathrm{GL}_{n}(\mathbb{C}): A J A^{-1}=\lambda J, \text { for some } \lambda \in \mathbb{C}^{*}\right\},
$$

where $J:=J_{n}(0)$ and it acts on $X$ as $A \cdot\left(N_{1}, N_{2}\right)=\left(A N_{1}, A N_{2}\right)$.

Lemma 3. The group $\mathcal{N}$ is the semidirect product

$$
\mathcal{N}=U \rtimes T \times Z,
$$

where $\mathcal{U}$ is the unipotent radical of $\mathcal{N}$ of unipotent lower triangular Toeplitz matrices, given by

$$
u:=\left\{\mathbf{1}_{n}+\sum_{i=1}^{n-1} a_{i} J_{n}^{i}(0): a_{i} \in \mathbb{C}\right\},
$$

$T$ is the one-dimensional torus $T=\left\{t_{\lambda}: \lambda \in \mathbb{C}^{*}\right\}$, where $t_{\lambda}$ is the diagonal matrix $t_{\lambda}:=\operatorname{diag}\left(1, \lambda, \lambda^{2}, \ldots, \lambda^{n-1}\right)$, and $Z$ consists of central elements $\left\{a_{0} \mathbf{1}_{n}: a_{0} \in \mathbb{C}^{*}\right\}$.

Proof. Every element of $\mathcal{U} T Z$ belongs to $\mathcal{N}$. Conversely, let $A \in \mathcal{N}$. Then it is easy to see that the columns $\boldsymbol{a}_{1}, \ldots, \boldsymbol{a}_{n}$ of $A$ satisfy the relation $\boldsymbol{a}_{k+1}=J \boldsymbol{a}_{k}$ and hence $A \in U T Z$. It is now easy to see that $U$ is normal in $U T Z$. 
For example for $n=5$, an element $A$ of the group $U$ and an element $t_{\lambda}$ of $T$ have the form

$$
A=\left(\begin{array}{ccccc}
1 & 0 & 0 & 0 & 0 \\
a_{1} & 1 & 0 & 0 & 0 \\
a_{2} & a_{1} & 1 & 0 & 0 \\
a_{3} & a_{2} & a_{1} & 1 & 0 \\
a_{4} & a_{3} & a_{2} & a_{1} & 1
\end{array}\right) t_{\lambda}=\left(\begin{array}{ccccc}
1 & 0 & 0 & 0 & 0 \\
0 & \lambda & 0 & 0 & 0 \\
0 & 0 & \lambda^{2} & 0 & 0 \\
0 & 0 & 0 & \lambda^{3} & 0 \\
0 & 0 & 0 & 0 & \lambda^{4}
\end{array}\right)
$$

for $a_{1}, a_{2}, a_{3}, a_{4} \in \mathbb{C}$ and $\lambda \in \mathbb{C}^{*}$.

Let $X^{T}:=\{N \in X: t N=N$ for all $t \in T\}$. Clearly $L \in X^{T}$ if and only if $L$ is a coordinate subrepresentation of $R_{n}$, i.e., both $L_{1}$ and $L_{2}$ are coordinate subspaces of $k^{n}$. In the next section we will encode this information in a combinatorial tool which is called the coefficient quiver of $R_{n}$.

We conclude this section by pointing out the useful isomorphism

$$
\varphi_{n}: \mathrm{Gr}_{\boldsymbol{e}} \rightarrow \mathrm{Gr}_{e^{*}}\left(R_{n}\right), \quad \varphi_{n}(N)=N^{0},
$$

where $\left(e_{1}, e_{2}\right)^{*}:=\left(n-e_{2}, n-e_{1}\right)$ and $N^{0}:=\left\{f \in R_{n}^{*}: f(v)=0\right.$ for all $\left.v \in N\right\}$ by using the identification $R_{n} \simeq R_{n}^{*}$. The torus $T$ acts on $R_{n}^{*}$ with contragredient action and this gives an action on $\operatorname{Gr}_{e^{*}}\left(R_{n}^{*}\right)$. Under the identification $R_{n} \simeq R_{n}^{*}$ these two actions differ by a character and so the identification $\operatorname{Gr}_{e}\left(R_{n}^{*}\right) \simeq \operatorname{Gr}_{e}\left(R_{n}\right)$ is $T$-equivariant. It is an easy check that isomorphism (10) is $T$-equivariant and involutive.

2.3. The coefficient quiver of $\boldsymbol{R}_{\boldsymbol{n}}$. Following [Ringel 1998] we associate to a $Q$ representation $M$ with linear basis $\mathscr{B}$ a quiver $\Gamma(M, \mathscr{B})$ called the coefficient quiver of $M$ in the basis $\mathscr{B}$. By definition, $\Gamma(M, \mathscr{B})$ has the elements of $\mathscr{B}$ as vertices and there is an arrow labeled by $a$ (resp. $b$ ) between two vertices $v$ and $v^{\prime}$ if the coefficient of $v^{\prime}$ in $m_{a}(v)$ (resp. $m_{b}(v)$ ) is nonzero. We call $\Gamma(M, \mathscr{B})$ the coefficient quiver of $M$ in the basis $\mathscr{B}$. When the basis $\mathscr{B}$ of $M$ is clear from the definition of $M$ we simply write $\Gamma(M)$ for $\Gamma(M, \mathscr{B})$. Here are the coefficient quivers of some indecomposable $Q$-representations for $n=4$ in the basis defined in Section 2:

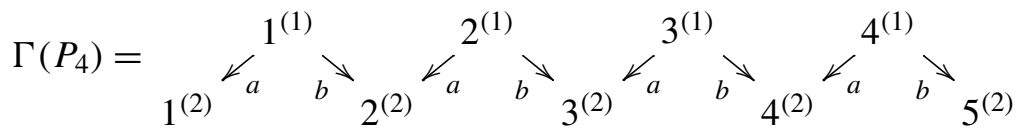

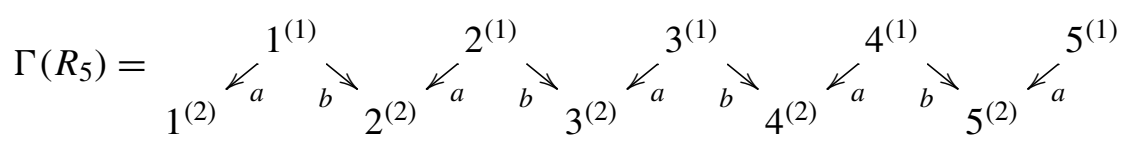

$$
\begin{aligned}
& \Gamma\left(I_{4}\right)=\quad 1^{(1)} \downarrow_{1^{(2)}} \swarrow_{a}^{2^{(1)}} b_{2^{(2)}} \swarrow_{a}^{3^{(1)}} b_{3^{(2)}}^{\downarrow_{a}}{ }_{b}^{4_{4}^{(2)}} \swarrow_{a}^{5^{(1)}}
\end{aligned}
$$


Here $k^{(i)}$, for $i=1,2$, denotes the vertex corresponding to $v_{k}^{(i)}$. Note that we use the convention (8).

In all these cases a one-dimensional torus $T$ acts on all the associated quiver Grassmannians and the fixed points of this action are in bijection with successor closed subquivers of the corresponding coefficient quiver (i.e., subquivers $\gamma$ such that if $v$ is a vertex of $\gamma$ and $\alpha: v \rightarrow v^{\prime}$ is an arrow with source $v$ then $\alpha$ is an arrow of $\gamma$ ).

Let us consider $\Gamma\left(R_{n}\right)$. In Lemma 3 we have seen that the torus $T$ acts on $X$ by $\lambda \cdot v_{k}^{(i)}=\lambda^{k-1} v_{k}^{(i)}(i=1,2, k \in[1, n])$. For every $r \geq 1$ there exists a unique regular subrepresentation of $R_{n}$ isomorphic to $R_{r}$ and it has the property that,

$$
\text { if } N \in X \text { is such that } \lim _{\lambda \rightarrow 0} t_{\lambda} N=R_{r} \text {, then } N=R_{r} \text {. }
$$

Indeed this subrepresentation is coordinate and lies in the extreme right-hand side of $\Gamma\left(R_{n}\right)$. In particular the basis elements that generate $R_{r}$ have maximal weights.

Roughly speaking, the flow for $\lambda \rightarrow 0$ goes from right to left in $\Gamma\left(R_{n}\right)$. For example the line $\left\langle v_{1}^{(2)}+v_{2}^{(2)}\right\rangle$ generated by the vector $v_{1}^{(2)}+v_{2}^{(2)}$ goes to the line generated by $v_{1}^{(2)}$ as follows:

$$
\lim _{\lambda \rightarrow 0} \lambda \cdot\left(\left\langle v_{1}^{(2)}+v_{2}^{(2)}\right\rangle\right)=\lim _{\lambda \rightarrow 0}\left(\left\langle v_{1}^{(2)}+\lambda v_{2}^{(2)}\right\rangle\right)=\left\langle v_{1}^{(2)}\right\rangle .
$$

2.4. Action of the torus on Hom-spaces. Let $L$ and $L^{\prime}$ be indecomposable $Q$ representations. As we have seen in the previous sections, the torus $T=\left\{t_{\lambda}\right.$ : $\left.\lambda \in \mathbb{C}^{*}\right\}$ acts on the quiver Grassmannians associated with them. The action of $T$ naturally extends to the vector space $\operatorname{Hom}\left(L, L^{\prime}\right)$ as follows: for $f \in \operatorname{Hom}\left(L, L^{\prime}\right)$, $\left(t_{\lambda} f\right)(l):=t_{\lambda} f\left(t_{\lambda^{-1}} l\right)$. Following [Crawley-Boevey 1989] we endow the vector space $\operatorname{Hom}\left(L, L^{\prime}\right)$ with a distinguished basis. Since $\operatorname{Hom}(\cdot, \cdot)$ is additive, we assume that both $L$ and $L^{\prime}$ are indecomposable. Let $\Gamma(L)$ and $\Gamma\left(L^{\prime}\right)$ denote the coefficient quiver of $L$ and $L^{\prime}$ respectively. We consider the set $\mathscr{G} \mathscr{B}\left(L, L^{\prime}\right)$ of triples $\left(\gamma, \alpha, \gamma^{\prime}\right)$ such that $\gamma$ is a connected predecessor closed subquiver of $\Gamma(L)$ (meaning that for every vertex $v$ of $\gamma$, every arrow $c: v^{\prime} \rightarrow v$ with target $v$ belongs to $\gamma), \gamma^{\prime}$ is a successor closed subquiver of $\Gamma\left(L^{\prime}\right)$, and $\alpha: \gamma_{0} \rightarrow \gamma_{0}^{\prime}$ is a bijection from the set $\gamma_{0}$ of vertices of $\gamma$ to the set $\gamma_{0}^{\prime}$ of vertices of $\gamma^{\prime}$, such that for every arrow

$$
v_{k}^{(1)} \stackrel{a}{\longrightarrow} v_{k}^{(2)}
$$

of $\gamma$ there is an arrow

$$
\alpha\left(v_{k}^{(1)}\right) \stackrel{a}{\longrightarrow} \alpha\left(v_{k}^{(2)}\right)
$$

of $\gamma^{\prime}$ with the same label, and likewise with $a$ replaced by $b$.

The next proposition is a special case of [Crawley-Boevey 1989], so we omit its proof (which is quite simple in this case). 
Proposition 4. Consider the map $B: \mathscr{C} \mathscr{B}\left(L, L^{\prime}\right) \rightarrow \operatorname{Hom}\left(L, L^{\prime}\right)$ that associates to $\left(\gamma, \alpha, \gamma^{\prime}\right)$ the homomorphism

$$
f_{\gamma \gamma^{\prime}}(v)=\left\{\begin{array}{cl}
\alpha(v) & \text { if } v \in \gamma_{0}, \\
0 & \text { otherwise. }
\end{array}\right.
$$

The image of $B$ is a basis of $\operatorname{Hom}\left(L, L^{\prime}\right)$ which we call $C B$-basis (for CrawleyBoevey).

The proposition is illustrated in the figure below, which shows an element of the $C B$-basis of $\operatorname{Hom}\left(R_{3}, R_{3}\right)$. The circles (resp. bullets) highlight a predecessor (successor) closed subquiver $\gamma$ (resp. $\gamma^{\prime}$ ) of $\Gamma\left(R_{3}\right)$. The dotted arrows show the corresponding $f_{\gamma \gamma^{\prime}}$.

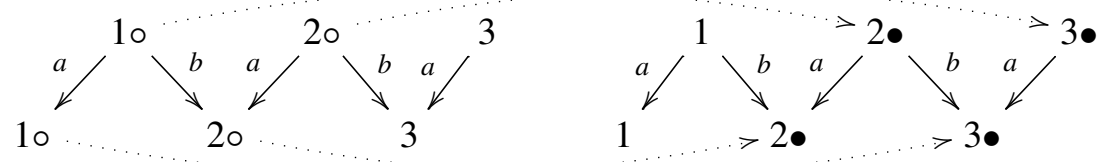

The torus $T$ acts diagonally on the elements of the $C B$-basis of $\operatorname{Hom}\left(L, L^{\prime}\right)$ as follows: If $\left(\gamma, \alpha, \gamma^{\prime}\right) \in \mathscr{C} \mathscr{B}\left(L, L^{\prime}\right)$ and the vertices of $\gamma$ have consecutive weights $k, k+1, \ldots$ and the vertices of $\gamma^{\prime}$ have consecutive weights $k^{\prime}, k^{\prime}+1, \ldots$ then

$$
t_{\lambda} f_{\gamma \gamma^{\prime}}=\lambda^{k^{\prime}-k} f_{\gamma \gamma^{\prime}}
$$

and we say that $f_{\gamma \gamma^{\prime}}$ has weight $k^{\prime}-k$. For example, the morphism illustrated above has weight 1 . We denote by $\operatorname{Hom}\left(L, L^{\prime}\right)^{+}$the vector subspace of $\operatorname{Hom}\left(L, L^{\prime}\right)$ spanned by $C B$-basis elements with positive weight.

As an application of Proposition 4 we compute the dimension of the Hom-spaces between indecomposable $Q$-representations. It is known (and not difficult to prove by using Proposition 4) that $\operatorname{Hom}\left(R_{s}, P_{l}\right)=\operatorname{Hom}\left(I_{s}, P_{l}\right)=\operatorname{Hom}\left(I_{s}, R_{l}\right)=0$ for all $s, l \geq 0$. Hence we consider the remaining cases.

Lemma 5. For every $l, s \geq 0$ we have

$$
\begin{aligned}
& \operatorname{dim} \operatorname{Hom}\left(P_{s}, P_{l}\right)=[l-s+1]_{+}, \\
& \operatorname{dim} \operatorname{Hom}\left(P_{s}, R_{l}\right)=l, \\
& \operatorname{dim} \operatorname{Hom}\left(P_{s}, I_{l}\right)=l+s, \\
& \operatorname{dim} \operatorname{Hom}\left(R_{s}, R_{l}\right)=\min (s, l), \\
& \operatorname{dim} \operatorname{Hom}\left(R_{s}, I_{l}\right)=s, \\
& \operatorname{dim} \operatorname{Hom}\left(I_{s}, I_{l}\right)=[s-l+1]_{+},
\end{aligned}
$$

where $[b]_{+}:=\max (b, 0)$. 
We conclude this section by pointing out that the action of the torus $T$ on $\operatorname{Hom}\left(L, L^{\prime}\right)$ induces an action of the torus on the space $\operatorname{Ext}^{1}\left(L, L^{\prime}\right)$. With respect to this action long exact sequences in cohomology are $T$-equivariant.

2.5. Stratification of $X$. Every subrepresentation $N$ of $R_{n}$ is of the form $N=$ $P \oplus R_{r}$ where $P$ is preprojective and $R_{r}$, for $r \geq 0$, is either zero or an indecomposable regular $Q$-representation. Similarly the quotient $R_{n} / N=R_{r^{\prime}} \oplus I$ where $R_{r^{\prime}}$, for $r^{\prime} \geq 0$, is either zero or regular indecomposable and $I$ is preinjective. This allows us to give the following definition.

Definition 6. Let $N \in X$ with $N=P \oplus R_{r}$ and $R_{n} / N=R_{r^{\prime}} \oplus I$ with $P$ preprojective, $I$ preinjective and some $r, r^{\prime} \geq 0$. We define the integer

$$
K_{N}=K_{N}(X):=\min \left(r, r^{\prime}\right)
$$

It is easy to see that $K_{N}=\operatorname{dim} \operatorname{Ext}^{1}\left(N, R_{n} / N\right)$. Indeed

$\operatorname{dim} \operatorname{Ext}^{1}\left(N, R_{n} / N\right)=\operatorname{dim} \operatorname{Ext}^{1}\left(R_{r}, R_{r^{\prime}}\right)=\operatorname{dim} \operatorname{Hom}\left(R_{r^{\prime}}, R_{r}\right)=\min \left(r, r^{\prime}\right)$

where in the first equality we use the well-known fact that

$$
\operatorname{Ext}^{1}(R, I)=\operatorname{Ext}^{1}(P, R)=\operatorname{Ext}^{1}(P, I)=0
$$

for every preprojective $P$, regular $R$ and preinjective $I Q$-representations; in the second equality we use the AR-formula (see [Assem et al. 2006], for example); in the last equality we use (15). In particular it is known that

$$
\left\langle\operatorname{dim} N, \operatorname{dim} N^{\prime}\right\rangle=\operatorname{dim} \operatorname{Hom}\left(N, N^{\prime}\right)-\operatorname{dim} \operatorname{Ext}^{1}\left(N, N^{\prime}\right)
$$

and in view of (6) we get that for every $N \in X$ the dimension of the tangent space $T_{N}(X)$ at $N$ equals

$$
\operatorname{dim} T_{N}(X)=\langle\boldsymbol{e}, n \delta-\boldsymbol{e}\rangle+K_{N} .
$$

where $\delta:=(1,1)^{t}$. Equation (18) implies that a point $N \in X$ is smooth if and only if either $N$ or $R_{n} / N$ do not have a regular direct summand.

The next theorem provides a stratification of $X$ and it is essential for our proof of the existence of a cellular decomposition of $X$. Let us define the strata.

Definition 7. For every integer $k \geq 0$ define the set

$$
X_{k}=X_{k}(X):=\left\{N \in X: K_{N} \geq k\right\}
$$

where $K_{N}$ is given in Definition 6.

Theorem 8. (1) The set $X_{k+1}$ is a closed T-stable subvariety of $X_{k}$. Moreover there is a $T$-equivariant isomorphism

$$
X_{k} \simeq \operatorname{Gr}_{\left(e_{1}-k, e_{2}-k\right)}\left(R_{n-2 k}\right) .
$$


(2) The subvarieties $X_{k}$ 's provide a stratification of $X$

$$
X=X_{0} \supset X_{1} \supset \cdots \supset X_{s}
$$

where $s=\min \left(e_{1}, n-e_{2}\right)$.

(3) The variety $X_{k} \backslash X_{k+1}$ is smooth (inside $X_{k}$ ) and

$$
\lim _{\lambda \rightarrow 0} t_{\lambda} N \in X_{k} \backslash X_{k+1} \quad \text { for all } N \in X_{k} \backslash X_{k+1} .
$$

Proof. We consider two subvarieties of $X$ :

$$
X_{k}^{\prime}(X):=\left\{N \in \operatorname{Gr}_{e}\left(R_{n}\right): R_{k} \text { is a subrepresentation of } N\right\}
$$

for $k \in\left[0, e_{1}\right]$, and

$$
X_{k}^{\prime \prime}(X):=\left\{N \in \operatorname{Gr}_{e}\left(R_{n}\right): R_{k} \text { is a quotient of } R_{n} / N\right\}
$$

for $k \in\left[0, n-e_{2}\right]$. It follows from the definitions that

$$
X_{k}=X_{k}^{\prime} \cap X_{k}^{\prime \prime} \text {. }
$$

We now collect some properties of $X_{k}^{\prime}$ and $X_{k}^{\prime \prime}$.

Lemma 9. (1) The isomorphism $\varphi_{n}$ defined in (10) induces an isomorphism

$$
X_{k}^{\prime}\left(\operatorname{Gr}_{e}\left(R_{n}\right)\right) \simeq X_{k}^{\prime \prime}\left(\operatorname{Gr}_{e^{*}}\left(R_{n}\right)\right),
$$

and hence also

$$
X_{k}^{\prime \prime}\left(\operatorname{Gr}_{e}\left(R_{n}\right)\right) \simeq X_{k}^{\prime}\left(\operatorname{Gr}_{e^{*}}\left(R_{n}\right)\right) .
$$

(2) For every $k \in\left[0, e_{1}\right], X_{k}^{\prime}(X)$ is a T-stable subvariety of $X$. For every $N \in$ $X_{k}^{\prime}(X), \lim _{\lambda \rightarrow 0} t_{\lambda} N \in X_{k}^{\prime}(X)$. There is a T-equivariant isomorphism

$$
X_{k}^{\prime}(X) \simeq \operatorname{Gr}_{e-k \delta}\left(R_{n-k}\right) .
$$

(3) For every $k \in\left[0, n-e_{2}\right], X_{k}^{\prime \prime}(X)$ is a $T$-stable subvariety of $X$. For every $N \in X_{k}^{\prime \prime}(X), \lim _{\lambda \rightarrow 0} t_{\lambda} N \in X_{k}^{\prime \prime}(X)$. There is a T-equivariant isomorphism

$$
X_{k}^{\prime \prime}(X) \simeq \operatorname{Gr}_{e}\left(R_{n-k}\right) .
$$

Proof. Part (1) is a straightforward check. Part (3) follows from part (1) by using the isomorphism (10). Part (2) follows from property (11) of $R_{k}$. It remains to check (24). We consider the map

$$
\Phi: X_{k}^{\prime}(X) \rightarrow \operatorname{Gr}_{e-k \delta}\left(R_{n-k}\right) \text { given by } \Phi(N)=\left(N / R_{k}\right) .
$$

Since there is a unique exact sequence of regular $Q$-representations

$$
0 \longrightarrow R_{k} \stackrel{\iota_{k}}{\longrightarrow} R_{n} \stackrel{\pi_{n-k}}{\longrightarrow} R_{n-k} \longrightarrow 0
$$

the map $\Phi$ is well defined and bijective. 
We now conclude the proof of Theorem 8. Everything follows from Lemma 9 by (23) except the smoothness of $X_{k} \backslash X_{k+1}$. To prove smoothness notice that it is sufficient to prove smoothness of $X_{0} \backslash X_{1}$ by (19), since $K_{N}\left(X_{k}\right)=K_{N}(X)-k$. By (18) $X_{0} \backslash X_{1}$ is the smooth locus of $X$.

Corollary 10. The variety $X=\operatorname{Gr}_{e}\left(R_{n}\right)$ is smooth if and only if $e_{1}=0$ (in which case $X \simeq \mathrm{Gr}_{e_{2}}\left(k^{n}\right)$ ) or $e_{2}=n$ (in which case $X \simeq \mathrm{Gr}_{e_{1}}\left(k^{n}\right)$ ).

Proof. By Theorem 8, $X$ is smooth if and only if $X=X_{0}$ if and only if $s=$ $\min \left(e_{1}, n-e_{2}\right)=0$.

Corollary 11. The quiver Grassmannian $\operatorname{Gr}_{\left(e_{1}, e_{2}\right)}\left(R_{n}\right)$ has dimension

$$
\operatorname{dim} \operatorname{Gr}_{\left(e_{1}, e_{2}\right)}\left(R_{n}\right)=\langle\boldsymbol{e}, n \delta-\boldsymbol{e}\rangle=\left(e_{2}-e_{1}\right)\left(n-\left(e_{2}-e_{1}\right)\right)
$$

Proof. In $X=\mathrm{Gr}_{e}\left(R_{n}\right)$ one can always find a smooth point, i.e., a subrepresentation $N$ of $R_{n}$ such that either $N$ or $R_{n} / N$ do not have a regular direct summand. It follows that $X_{0} \backslash X_{1}$ is nonempty and hence the result follows from (18).

2.6. Cellular decomposition of $X$. In this section we provide a cellular decomposition of $X=\operatorname{Gr}_{e}\left(R_{n}\right)$. Following [Białynicki-Birula 1973] (see also [Chriss and Ginzburg 1997]), for every fixed point $L \in X^{T}$ we consider its attracting set, defined as

$$
X_{L}:=\left\{N \in X: \lim _{\lambda \rightarrow 0} t_{\lambda} N=L\right\} .
$$

In particular, $L \in X_{L}$ for every $L \in X^{T}$.

Theorem 12. For every $L \in X^{T}$ the corresponding attracting set $X_{L}$ is an affine space and $X_{L} \simeq T_{L}\left(X_{L}\right) \simeq T_{L}^{+}(X):=\operatorname{Hom}\left(L, R_{n} / L\right)^{+}$. Moreover

$$
X=\bigcup_{L \in X^{T}} X_{L}
$$

Proof. The results from [Białynicki-Birula 1973] on cellular decomposition of a projective variety $X$ continue to hold if the variety is smooth but only quasiprojective, provided that the action of the torus is such that $\lim _{\lambda \rightarrow 0} t_{\lambda} N$ belongs to $X$ for every $N \in X$. This is an easy consequence of Hironaka's resolution of singularities. In view of Theorem 8 we apply this to $X_{0} \backslash X_{1}$ and we get the result.

2.7. Description of the cells. In this section we describe the cell $X_{L}$ associated with every $L \in X^{T}$ (see Theorem 12). Given integers $r \in[0, n-1]$ and $k \in[1, n-r]$, we denote by ${ }_{k}\left(P_{r}\right)$ the indecomposable preprojective subrepresentation of $R_{n}$ of dimension vector $(r, r+1)$ generated by $v_{k}^{(1)}, v_{k+1}^{(1)}, \ldots, v_{k+r-1}^{(1)}$ and $v_{k}^{(2)}, v_{k+1}^{(2)}, \ldots, v_{k+r}^{(2)}$ if $r \geq 1$ and by $v_{k}^{(2)}$ if $r=0$. For example, the following figure shows the subrepre- 
sentation $_{2}\left(P_{1}\right)$ of $R_{5}$ :

$1^{(2)}$
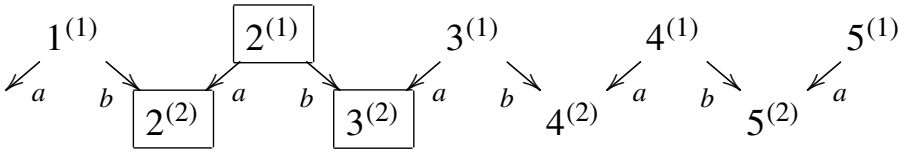

Theorem 13. (1) If $L \in X^{T}$ is indecomposable then $X_{L}=U L$. In particular if $L={ }_{k}\left(P_{e_{1}}\right)$ then $\operatorname{dim} X_{L}=n-k$.

(2) If $L=L^{\prime} \oplus L^{\prime \prime}$ with $\operatorname{Hom}\left(L^{\prime}, L^{\prime \prime}\right)^{+}=\operatorname{Hom}\left(L^{\prime}, L^{\prime \prime}\right)$ then

$$
\operatorname{dim} X_{L}=\operatorname{dim} X_{L^{\prime}}+\operatorname{dim} X_{L^{\prime \prime}}-\left\langle\operatorname{dim} L^{\prime}, \operatorname{dim} L^{\prime \prime}\right\rangle .
$$

Proof. (1) The unipotent group $U$ defined in Lemma 3 is a subgroup of dimension $n-1$ of the group of $n \times n$ unipotent lower triangular matrices. Let $L$ be an indecomposable subrepresentation of $R_{n}$ of dimension vector $\boldsymbol{e}$. If $L=R_{r}$ then $\boldsymbol{e}=(r, r)$ and $L_{1}=L_{2}$ is the vector subspace of $k^{n}$ spanned by the last $r$ basis vectors of $R_{n}$. In particular, $A L=L$ for every $A \in U$. On the other hand we have already noticed that regular subrepresentations have property (11) and hence $X_{L}=\{L\}$ and we get $X_{L}=U L$ if $L$ is regular. Let $L$ be an indecomposable preprojective subrepresentation of $R_{n}$. Then $e_{2}=e_{1}+1$ and we assume that $L={ }_{k}\left(P_{e_{1}}\right)$ for some $k \in\left[1, n-e_{1}\right]$. It is easy to see that $\operatorname{dim} U L=n-k$. Indeed the stabilizer of $L$ under the action of $U$ equals the stabilizer of the line generated by $v_{k}^{(2)}$ and hence it has dimension $k-1$. For example in (9) the stabilizer of the third basis vector is generated by $a_{3}$ and $a_{4}$ and has dimension two. We now prove that $\operatorname{dim} X_{L}=n-k$ and since $U L \subset X_{L}$ and they are both affine spaces, we get the equality. By Theorem 12 we have to compute $\operatorname{dim} T_{L}^{+}(X)=\operatorname{dim} \operatorname{Hom}\left(L, R_{n} / L\right)^{+}$. Since $L={ }_{k}\left(P_{e_{1}}\right)$ is indecomposable the quotient $R_{n} / L$ is the direct sum of at most two indecomposables as follows

$$
R_{n} / L=R_{k-1} \oplus I_{t}
$$

where $t=n-k-e_{1}$. We have

$$
\operatorname{Hom}\left(L, R_{n} / L\right)^{+}=\operatorname{Hom}\left(P_{e_{1}}, I_{t}\right) .
$$

In view of (14), $\operatorname{dim} \operatorname{Hom}\left(L, R_{n} / L\right)^{+}=e_{1}+t=n-k$ and we are done.

(2) There are short exact sequences

$$
0 \longrightarrow L^{\prime} \longrightarrow R_{n} / L^{\prime \prime} \longrightarrow R_{n} /\left(L^{\prime} \oplus L^{\prime \prime}\right) \longrightarrow 0
$$

and

$$
0 \longrightarrow L^{\prime \prime} \longrightarrow R_{n} / L^{\prime} \longrightarrow R_{n} /\left(L^{\prime} \oplus L^{\prime \prime}\right) \longrightarrow 0
$$


We apply the functors $\operatorname{Hom}\left(L^{\prime \prime},-\right)$ and $\operatorname{Hom}\left(L^{\prime},-\right)$ to the previous short exact sequences, then take the positive part (the part spanned by $C B$-basis elements with positive weight) and we get the exact sequences (see Theorem 12)

$$
0 \longrightarrow 0 \longrightarrow T_{L^{\prime \prime}}\left(X_{L^{\prime \prime}}\right) \longrightarrow \operatorname{Hom}\left(L^{\prime \prime}, R_{n} / L\right) \longrightarrow 0
$$

and

$$
0 \rightarrow \operatorname{Hom}\left(L^{\prime}, L^{\prime \prime}\right) \rightarrow T_{L^{\prime}}\left(X_{L^{\prime}}\right) \rightarrow \operatorname{Hom}\left(L^{\prime}, R_{n} / L\right) \rightarrow \operatorname{Ext}^{1}\left(L^{\prime}, L^{\prime \prime}\right)^{+} \rightarrow 0 .
$$

Indeed, we have

$$
\begin{aligned}
& \operatorname{Ext}^{1}\left(L^{\prime \prime}, L^{\prime}\right)^{+}=0=\operatorname{Ext}^{1}\left(L^{\prime}, R_{n} / L^{\prime}\right)^{+}, \\
& \operatorname{Ext}^{1}\left(L^{\prime}, L^{\prime \prime}\right)^{+}=\operatorname{Ext}^{1}\left(L^{\prime}, L^{\prime \prime}\right) .
\end{aligned}
$$

This is because, if $\operatorname{Hom}(M, L)^{+}=0$ then $\operatorname{Ext}^{1}(M, L)^{+}=0$; indeed one can always take a minimal $T$-equivariant injective resolution of $L$ and apply the functor $\operatorname{Hom}(M,-)$. By summing up we get the short exact sequence

$$
0 \rightarrow \operatorname{Hom}\left(L^{\prime}, L^{\prime \prime}\right) \rightarrow T_{L^{\prime}}\left(X_{L^{\prime}}\right) \oplus T_{L^{\prime \prime}}\left(X_{L^{\prime \prime}}\right) \rightarrow T_{L}\left(X_{L}\right) \rightarrow \operatorname{Ext}^{1}\left(L^{\prime}, L^{\prime \prime}\right) \rightarrow 0
$$

Since for every fixed point $L, \operatorname{dim} X_{L}=\operatorname{dim} T_{L}\left(X_{L}\right)$ we get (28).

2.8. Betti numbers. We now use the results of the previous sections in order to compute the Betti numbers of $X=\operatorname{Gr}_{e}\left(R_{n}\right)$. Since $X$ has a cellular decomposition (Theorem 12) the odd cohomology spaces of $X$ are zero and the $2 i$-th Betti number $b_{2 i}=b_{2 i}(X):=\operatorname{dim} H^{2 i}(X)$ equals the number of cells of dimension $i$.

Before stating the main result of this section we start with the special case $e_{2}=e_{1}+1$.

Theorem 14. Let $X=\operatorname{Gr}_{\left(e_{1}, e_{1}+1\right)}\left(R_{n}\right)$. The even Betti numbers of $X$ are

$$
b_{2 i}= \begin{cases}i+1 & \text { if } 0 \leq i \leq s \\ s+1 & \text { if } s \leq i \leq n-1-s \\ n-i & \text { if } n-1-s \leq i \leq n-1\end{cases}
$$

where $s=\min \left(e_{1}, n-1-e_{1}\right)($ see Theorem 8(2)).

The Poincaré polynomial $P_{X}(t):=\sum_{i=0}^{\operatorname{dim} X} b_{i} t^{i}$ of $X$ equals

$$
P_{X}\left(t^{1 / 2}\right)=\left(\frac{t^{e_{1}+1}-1}{t-1}\right)\left(\frac{t^{n-e_{1}}-1}{t-1}\right)
$$

Proof. For $k \in\left[0, e_{1}\right]$, consider the variety $X_{k}^{\prime}$ defined in (21) and the difference

$$
Y_{k}^{\prime}=Y_{k}^{\prime}(X):=X_{k}^{\prime}(X) \backslash X_{k+1}^{\prime}(X) \text {. }
$$


By definition, $N \in Y_{k}$ if $N=R_{k} \oplus P$ for some (indecomposable) preprojective $P$. Since $X$ is union of the $Y_{k}^{\prime}$ and each $Y_{k}^{\prime}$ is a union of cells (Theorem 12) we get

$$
\operatorname{dim} H^{i}(X)=\sum_{k=0}^{e_{1}} \operatorname{dim} H_{c}^{i}\left(Y_{k}^{\prime}\right),
$$

where $H_{c}^{i}\left(Y_{k}^{\prime}\right)$ is the $i$-th cohomology space of $Y_{k}^{\prime}$ with compact support and its dimension equals the number of cells of dimension $i / 2$. In particular $\operatorname{dim} H_{c}^{i}\left(Y_{k}^{\prime}\right)=0$ for $i$ odd. We prove that

$$
\operatorname{dim} H_{c}^{2 i}\left(Y_{k}^{\prime}\right)= \begin{cases}1 & \text { if } i \in\left[e_{1}-k, n-k-1\right], \\ 0 & \text { otherwise. }\end{cases}
$$

and hence (31) follows from (34). From the definition it follows that

$$
Y_{k}^{\prime}(X) \simeq Y_{0}^{\prime}\left(\operatorname{Gr}_{\left(e_{1}-k, e_{1}+1-k\right)}\left(R_{n-k}\right)\right) .
$$

The elements of $Y_{0}^{\prime}\left(\mathrm{Gr}_{\left(f_{1}, f_{1}+1\right)}\left(R_{m}\right)\right)$ are all the indecomposable preprojective subrepresentations of $R_{m}$ of dimension vector $\left(f_{1}, f_{1}+1\right)$ and hence they are all isomorphic to $P_{f_{1}}$. For $i \in\left[1, m-f_{1}\right]$, Theorem 13(1) gives $\operatorname{dim} X_{i\left(P_{f_{1}}\right)}=m-i$, which is in $\left[f_{1}, m-1\right]$; hence for every $\ell \in\left[f_{1}, m-1\right]$ there is a unique cell of dimension $\ell$. For $m=n-k$ and $f_{1}=e_{1}-k$ we get (35).

Notice that (32) can be written as $P_{X}(t)=P_{\mathrm{Gr}_{1}\left(e_{2}\right)}(t) P_{\mathrm{Gr}_{1}\left(n-e_{1}\right)}(t)$, where $e_{2}=$ $e_{1}+1$. Surprisingly, this turns out to be a general fact, which we state as the main result of this section:

Theorem 15. Let $X=\operatorname{Gr}_{\left(e_{1}, e_{2}\right)}\left(R_{n}\right)$. The Poincaré polynomial $P_{X}(t)$ of $X$ equals

$$
P_{X}(t)=P_{\mathrm{Gr}_{\left(e_{2}-e_{1}\right)}\left(e_{2}\right)}(t) P_{\mathrm{Gr}_{\left(e_{2}-e_{1}\right)}\left(n-e_{1}\right)}(t) .
$$

Proof. We proceed by induction on $n \geq e_{1} \geq 0$. For $e_{1}=0, X \simeq \operatorname{Gr}_{e_{2}}(n)$ and (37) follows. Let $1 \leq e_{1} \leq e_{2}$. The variety $X$ can be decomposed as

$$
X=Y_{0}^{\prime}(X) \cup X_{1}^{\prime}(X)
$$

where $Y_{0}^{\prime}=Y_{0}^{\prime}(X)$ consists of all preprojective subrepresentations of $R_{n}$ in $X$ and $X_{1}^{\prime}$ consists of subrepresentations of $R_{n}$ in $X$ having a nonzero regular subrepresentation (see (21) and (33)). Moreover, by (24), $X_{1}^{\prime}(X) \simeq \operatorname{Gr}_{\left(e_{1}-1, e_{2}-1\right)}\left(R_{n-1}\right)$ and hence we have

$$
P_{X}(t)=P_{Y_{0}^{\prime}}(t)+P_{\operatorname{Gr}_{\left(e_{1}-1, e_{2}-1\right)}\left(R_{n-1}\right)}(t) .
$$

By the inductive hypothesis (37) holds if and only if

$$
P_{Y_{0}^{\prime}}(t)=\left(P_{\mathrm{Gr}_{\left(e_{2}-e_{1}\right)}\left(e_{2}\right)}(t)-P_{\mathrm{Gr}_{\left(e_{2}-e_{1}\right)}\left(e_{2}-1\right)}(t)\right) P_{\mathrm{Gr}_{\left(e_{2}-e_{1}\right)}\left(n-e_{1}\right)}(t) .
$$

Hence we prove (39). 
We make the following choice: we fix a linear basis $\left\{v_{1}, \ldots, v_{s}\right\}$ of a vector space of dimension $s$ and we let the torus act on $\mathrm{Gr}_{t}(s)$ by $t_{\lambda} v_{i}=\lambda^{i} v_{i}$. We consider the vector subspace of $k^{e_{2}}$ generated by $v_{2}, \ldots, v_{e_{2}}$ and the corresponding embedding $\operatorname{Gr}_{\left(e_{2}-e_{1}\right)}\left(e_{2}-1\right) \subset \operatorname{Gr}_{\left(e_{2}-e_{1}\right)}\left(e_{2}\right)$. With this choice the difference $\operatorname{Gr}_{\left(e_{2}-e_{1}\right)}\left(e_{2}\right) \backslash$ $\operatorname{Gr}_{\left(e_{2}-e_{1}\right)}\left(e_{2}-1\right)$ is $T$-stable and for every point $W$ of it, $\lim _{\lambda \rightarrow 0} t_{\lambda} W$ still belongs to it. The right-hand side of (39) is the Poincaré polynomial (with respect to the cohomology with compact support) of the smooth projective variety

$$
G:=\left(\operatorname{Gr}_{\left(e_{2}-e_{1}\right)}\left(e_{2}\right) \backslash \operatorname{Gr}_{\left(e_{2}-e_{1}\right)}\left(e_{2}-1\right)\right) \times \operatorname{Gr}_{\left(e_{2}-e_{1}\right)}\left(n-e_{1}\right) .
$$

The one-dimensional torus $T$ acts on $G$ and the attracting sets of the $T$-fixed points form a cellular decomposition of $G$. We prove that there exists a bijection between the cells of $Y_{0}^{\prime}$ of dimension $k$ and the cells of $G$ of dimension $k$.

A point of $Y_{0}^{\prime}$ is a direct sum of precisely $\left(e_{2}-e_{1}\right)$ indecomposable preprojective subrepresentations of $R_{n}$ (this follows by considering their dimension vectors). The $T$-fixed points have the form

$$
\left.L:={ }_{k_{1}}\left(P_{r_{1}}\right) \oplus_{k_{2}}\left(P_{r_{2}}\right) \oplus \cdots \oplus{k_{\left(e_{2}-e_{1}\right)}}_{r_{\left(e_{2}-e_{1}\right)}}\right),
$$

where $r_{1}+\cdots+r_{e_{2}-e_{1}}=e_{1}, r_{i} \geq 0$ and ${ }_{k}\left(P_{r}\right)$ has the same meaning as in Section 2.7. In view of Theorem 13 and of (12) the dimension of the attracting cell of $L$ equals

$$
\operatorname{dim} X_{L}=n\left(e_{2}-e_{1}\right)-\sum_{i=1}^{e_{2}-e_{1}} k_{i}-\left(e_{2}-e_{1}\right)^{2}+\sum_{i=1}^{e_{2}-e_{1}} \sum_{j=1}^{i}\left(r_{j}-r_{i}+1\right) .
$$

We consider the set $\alpha(\boldsymbol{e}, k, n)$ which parametrizes the $T$-fixed points of $Y_{0}^{\prime}$ whose attracting set has dimension $k$, i.e.,

$$
\begin{aligned}
\alpha(\boldsymbol{e}, k, n):=\{ & \left(k_{1}, k_{2}, \ldots, k_{e_{2}-e_{1}}, r_{1}, r_{2}, \ldots, r_{e_{2}-e_{1}}\right): \\
& 1 \leq k_{1} \leq k_{1}+r_{1}<k_{2} \leq k_{2}+r_{2}<\cdots<k_{e_{2}-e_{1}} \leq k_{e_{2}-e_{1}}+r_{e_{2}-e_{1}} \leq n, \\
& r_{1}+r_{2}+\cdots+r_{e_{2}-e_{1}}=e_{1}, r_{i} \geq 0, \\
& \left.n\left(e_{2}-e_{1}\right)-\sum_{i=1}^{e_{2}-e_{1}} k_{i}-\left(e_{2}-e_{1}\right)^{2}+\sum_{i=1}^{e_{2}-e_{1}} \sum_{j=1}^{i}\left(r_{j}-r_{i}+1\right)=k\right\} .
\end{aligned}
$$

Now consider the $T$-fixed points of $G$ and their attracting sets. The $T$-fixed points of $\mathrm{Gr}_{t}(s)$ consist of coordinate vector subspaces of dimension $t$ and they are naturally parametrized by tuples $\left(a_{1}, \ldots, a_{t}\right)$ of integers $1 \leq a_{1}<\cdots<a_{t} \leq s$. The corresponding cell $\mathrm{O}_{\left(a_{1}, \ldots, a_{t}\right)}$ has dimension

$$
s-a_{1}-(t-1)+s-a_{2}-(t-2)+\cdots s-a_{t}=t s-\sum_{i=1}^{t} a_{i}-\sum_{i=1}^{t-1} i .
$$

The $T$-fixed points of $\operatorname{Gr}_{\left(e_{2}-e_{1}\right)}\left(e_{2}\right) \backslash \mathrm{Gr}_{\left(e_{2}-e_{1}\right)}\left(e_{2}-1\right)$ are the coordinate vector subspaces of $k^{e_{2}}$ containing $v_{1}$. Hence the following set parametrizes the cells of $G$ 
of dimension $k$ :

$$
\begin{gathered}
\beta(\boldsymbol{e}, k, n):=\left\{\left(a_{1}, a_{2}, \ldots, a_{e_{2}-e_{1}}, b_{2}, b_{3}, \ldots, b_{e_{2}-e_{1}}\right):\right. \\
1 \leq a_{1}<a_{2}<\cdots<a_{e_{2}-e_{1}} \leq n-e_{1}, \quad 2 \leq b_{2}<\cdots<b_{e_{2}-e_{1}} \leq e_{2}, \\
\left.n\left(e_{2}-e_{1}\right)-\sum_{i=1}^{e_{2}-e_{1}} a_{i}-\sum_{i=2}^{e_{2}-e_{1}} b_{i}+\left(e_{2}-e_{1}-1\right)=k\right\} .
\end{gathered}
$$

We consider the map $\beta(\boldsymbol{e}, k, n) \rightarrow \alpha(\boldsymbol{e}, k, n)$ defined by

$$
a_{1} \mapsto k_{1}, \quad a_{i} \mapsto k_{i}-r_{1}-r_{2}-\cdots-r_{i-1}, \quad b_{i} \mapsto \sum_{j=0}^{i-2} r_{\left(e_{2}-e_{1}-j\right)}+i,
$$

for $i \in\left[2, e_{2}-e_{1}\right]$. It is straightforward to verify that this map is a bijection between $\beta(\boldsymbol{e}, k, n)$ and $\alpha(\boldsymbol{e}, k, n)$. It follows that $Y_{0}^{\prime}$ and $G$ have the same Betti numbers and hence (39) follows.

Corollary 16. The Poincaré polynomial of a quiver Grassmannian associated with the indecomposable preprojective $P_{n}$ and the indecomposable preinjective $I_{n}$, where $n \geq 0$, are given by

$$
\begin{aligned}
& P_{\mathrm{Gr} e\left(P_{n}\right)}(t)=P_{\mathrm{Gr}_{e_{1}}\left(e_{2}-1\right)}(t) P_{\mathrm{Gr}_{\left(e_{2}-e_{1}\right)}\left(n+1-e_{1}\right)}(t), \\
& P_{\mathrm{Gr}_{e}\left(I_{n}\right)}(t)=P_{\mathrm{Gr}_{e_{1}}\left(e_{2}+1\right)}(t) P_{\mathrm{Gr}_{\left(e_{2}-e_{1}\right)}\left(n-e_{1}\right)}(t) .
\end{aligned}
$$

Proof. Equality (41) follows from (40) by the isomorphism

$$
\operatorname{Gr}_{\left(e_{1}, e_{2}\right)}\left(I_{n}\right) \simeq \operatorname{Gr}_{\left(n-e_{2}, n+1-e_{1}\right)}\left(P_{n}\right) .
$$

Hence we prove (40). As in the proof of Theorem 15, let $Y_{0}^{\prime}=Y_{0}^{\prime}\left(\operatorname{Gr}_{e}\left(R_{n+1}\right)\right)$ be the subvariety of $\operatorname{Gr}_{e}\left(R_{n+1}\right)$ of all preprojective subrepresentations of $R_{n+1}$ of dimension vector $\boldsymbol{e}$ and let

$$
G^{\prime}:=\operatorname{Gr}_{\left(e_{2}-e_{1}\right)}\left(e_{2}\right) \backslash \operatorname{Gr}_{\left(e_{2}-e_{1}\right)}\left(e_{2}-1\right)
$$

with the convention that $\operatorname{Gr}_{\left(e_{2}-e_{1}\right)}\left(e_{2}-1\right)$ consists of all the elements of $\operatorname{Gr}_{\left(e_{2}-e_{1}\right)}\left(e_{2}\right)$ not containing the first basis vector (as in the proof of Theorem 15). In view of (39) it is sufficient to prove the equalities

$$
\begin{aligned}
& P_{G^{\prime}}(t)=t^{2 e_{1}} P_{\operatorname{Gr}_{\left(e_{2}-e_{1}-1\right)}\left(e_{2}-1\right)}(t), \\
& P_{Y_{0}^{\prime}}(t)=t^{2 e_{1}} P_{\operatorname{Gr}_{e}\left(P_{n}\right)}(t) .
\end{aligned}
$$

The proof of (42) is similar to the proof of Theorem 15: there is an obvious bijection between the cells of $G^{\prime}$ of dimension $k$ and the cells of $\operatorname{Gr}_{\left(e_{2}-e_{1}\right)}\left(e_{2}-1\right)$ of dimension $k-e_{1}$. Let us prove (43). Let $L \in Y_{0}^{\prime}$. Then $L$ is a sum of preprojective subrepresentations of $R_{n+1}$ and $L$ is a subrepresentation of $P_{n} \leq_{Q} R_{n+1}$. By looking at the quotients $R_{n+1} / L$ and $P_{n} / L$ and using Lemma 5 one gets

$$
\operatorname{dim} \operatorname{Hom}\left(L, R_{n+1} / L\right)^{+}=\operatorname{dim} \operatorname{Hom}\left(L, P_{n} / L\right)^{+}+e_{1} .
$$


Hence the cells of $Y_{0}^{\prime}$ of dimension $k$ are in bijection with the cells of $\operatorname{Gr}_{e}\left(P_{n}\right)$ of dimension $k-e_{1}$ and (43) holds.

Since $\chi(X)=P_{X}(1)$ for a projective variety $X$ and $\chi\left(\mathrm{Gr}_{t}(s)\right)=\left(\begin{array}{l}s \\ t\end{array}\right)$, we recover the following result, of which alternative proofs have also appeared in [Cerulli Irelli 2011b; Zelevinsky 2007; Szanto 2011; Poettering 2010].

Corollary 17 [Caldero and Zelevinsky 2006].

$$
\begin{aligned}
\chi\left(\operatorname{Gr}_{e}\left(R_{n}\right)\right) & =\left(\begin{array}{c}
e_{2} \\
e_{1}
\end{array}\right)\left(\begin{array}{c}
n-e_{1} \\
e_{2}-e_{1}
\end{array}\right), \\
\chi\left(\operatorname{Gr}_{e}\left(P_{n}\right)\right) & =\left(\begin{array}{c}
e_{2}-1 \\
e_{1}
\end{array}\right)\left(\begin{array}{c}
n+1-e_{1} \\
e_{2}-e_{1}
\end{array}\right), \\
\chi\left(\operatorname{Gr}_{e}\left(I_{n}\right)\right) & =\left(\begin{array}{c}
e_{2}+1 \\
e_{1}
\end{array}\right)\left(\begin{array}{c}
n-e_{1} \\
e_{2}-e_{1}
\end{array}\right) .
\end{aligned}
$$

\section{Applications to cluster algebras}

To a finite quiver $Q$ without loops and 2-cycles is associated a (coefficient-free) cluster algebra $\mathscr{A}_{Q}$ ([Fomin and Zelevinsky 2002]; see also [Fomin and Zelevinsky 2003b] and [Keller 2010] for excellent surveys). This is a $\mathbb{Z}$-subalgebra of the field $\mathscr{F}_{\mathrm{F}}$ of rational functions in $n$ variables ( $n$ being the number of vertices of $Q$ ), generated by its cluster variables. The cluster variables are grouped into free-generating sets of $\mathscr{F}$ called clusters. The cluster monomials are monomials in cluster variables belonging to the same cluster. An atomic basis of $\mathscr{A}_{Q}$ is a $\mathbb{Z}$-basis $\boldsymbol{B}$ of it such that the positive linear combinations of elements of $\boldsymbol{B}$ coincide with the semiring of positive elements of $\mathscr{A}_{Q}$ (elements that are positive Laurent polynomials in every cluster of $\mathscr{A}_{Q}$ ); see [Sherman and Zelevinsky 2004]. The existence of such a basis has been proved only in a few cases:

- If $Q$ is of type $A D E$, cluster monomials form an atomic basis of $\mathscr{A}_{Q}$ [Cerulli Irelli 2011a; Cerulli Irelli and Labardini-Fragoso 2011].

- When $Q$ is the Kronecker quiver, atomic bases exist coefficients and consist of cluster monomials together with extra elements $\left\{z_{n}: n \geq 1\right\}$ [Sherman and Zelevinsky 2004].

- when $Q$ is of type $A_{2}^{(1)}$, the atomic bases of $\mathscr{A}_{Q}$ have been found in [Cerulli Irelli 2009] (for every choice of the coefficients) and consist of cluster monomials together with some extra elements $\left\{u_{n}: n \geq 1\right\}$ possibly multiplied by particular cluster variables.

We notice that under the Caldero-Keller bijection, cluster monomials correspond, via the Caldero-Chapoton map, to rigid $Q$-representations and the quiver Grassmannians associated with rigid representations are smooth. In the next two sections 
we propose a "truncation" of the Caldero-Chapoton map that gives a geometric realization of the extra elements $\left\{z_{n}\right\},\left\{u_{n}\right\}$.

3.1. Type $\boldsymbol{A}_{1}^{(\mathbf{1})}$. Let $\mathscr{F}=\boldsymbol{Q}\left(x_{1}, x_{2}\right)$ be the field of rational functions in two independent variables $x_{1}$ and $x_{2}$ with rational coefficients. We recursively define elements $\left\{x_{k}: k \in \mathbb{Z}\right\}$ of $\mathscr{F}$ by

$$
x_{k} x_{k+2}=x_{k+1}^{2}+1, \quad k \in \mathbb{Z} .
$$

Let $\mathscr{A}$ be the $\mathbb{Z}$-subalgebra of $\mathscr{F}$ generated by all the $x_{k}$ 's $(k \in \mathbb{Z})$. By [Sherman and Zelevinsky 2004] the algebra $\mathscr{A}$ is the coefficient-free cluster algebra associated with the Kronecker quiver. The pairs $\left\{x_{k}, x_{k+1}\right\}, k \in \mathbb{Z}$, are free generating sets of $\mathscr{F}$ and form the clusters of $\mathscr{A}$. Monomials $x_{k}^{a} x_{k+1}^{b}, a, b \geq 0, k \in \mathbb{Z}$, are called the cluster monomials of $\mathscr{A}$.

Caldero and Zelevinsky [2006] defined the rational function

$$
s_{n}:=C C\left(R_{n}\right)
$$

for every $n \geq 1$, where $R_{n}$ is a regular indecomposable $Q$-representation of dimension $(n, n)$. They have proved that the set $\mathscr{S}:=$ cluster monomials $\} \cup\left\{s_{n}: n \geq 1\right\}$ is a $\mathbb{Z}$-basis of $\mathscr{A}$.

The atomic basis $\boldsymbol{B}$ defined in the introduction and the basis $\mathscr{S}$ are related by (see [Caldero and Zelevinsky 2006])

$$
z_{n}=s_{n}-s_{n-2}
$$

for $n \geq 1$ and the convention that $s_{-k}=0$ for $k>0, s_{0}:=1$.

Theorem 18. The element $z_{n}$ has the Laurent expansion

$$
z_{n}=\frac{\sum_{\boldsymbol{e}} \chi\left(\mathrm{Gr}_{\boldsymbol{e}}\left(R_{n}\right)^{S m}\right) x_{1}^{2\left(n-e_{2}\right)} x_{2}^{2 e_{1}}}{x_{1}^{n} x_{2}^{n}}
$$

where $\mathrm{Gr}_{\boldsymbol{e}}\left(R_{n}\right)^{\text {Sm }}$ denotes the smooth part of $\mathrm{Gr}_{\boldsymbol{e}}\left(R_{n}\right)$.

Proof. In view of Theorem $8, \operatorname{Gr}_{e}\left(R_{n}\right)^{S m}=X_{0} \backslash X_{1}$ where $X_{0}=\operatorname{Gr}_{e}\left(R_{n}\right)$ and $X_{1}=\operatorname{Gr}_{\left(e_{1}-1, e_{2}-1\right)}\left(R_{n-2}\right)$ and hence

$$
\chi\left(\operatorname{Gr}_{\boldsymbol{e}}\left(R_{n}\right)^{S m}\right)=\chi\left(\mathrm{Gr}_{\boldsymbol{e}}\left(R_{n}\right)\right)-\chi\left(\operatorname{Gr}_{\left(e_{1}-1, e_{2}-1\right)}\left(R_{n-2}\right)\right) .
$$

It is now easy to check that the right-hand side of (48) satisfies (47).

3.2. Type $\boldsymbol{A}_{2}^{(\mathbf{1})}$. We now briefly recall the construction of the atomic basis of cluster algebras of type $A_{2}^{(1)}$ from [Cerulli Irelli 2009]. Let $\mathscr{F}=\mathbb{Q}\left(x_{1}, x_{2}, x_{3}\right)$ be the field of rational functions in three (commuting) independent variables $x_{1}, x_{2}$ and $x_{3}$ with rational coefficients. Recursively define elements $x_{m} \in \mathscr{F}$ for $m \in \mathbb{Z}$ by the relation

$$
x_{m} x_{m+3}=x_{m+1} x_{m+2}+1 .
$$


Define also the elements $w, z \in \mathscr{F}$ by

$$
w=\frac{x_{1}+x_{3}}{x_{2}}, \quad z=\frac{x_{1} x_{2}+x_{2} x_{3}+1}{x_{1} x_{3}} .
$$

The (coefficient-free) cluster algebra $\mathscr{A}$ of type $A_{2}^{(1)}$ is the $\mathbb{Z}$-subalgebra of $\mathscr{F}$ generated by all the $x_{m}$ 's, $w$ and $z$ (see also [Fomin and Zelevinsky 2002, Example 7.8]). This is the cluster algebra $\mathscr{A}=\mathscr{A}_{Q_{2}}$ associated with the affine quiver

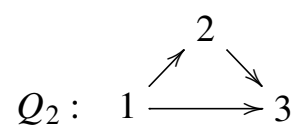

of type $A_{2}^{(1)}$. The elements $x_{m}, m \in \mathbb{Z}, w$ and $z$ are the cluster variables of $\mathscr{A}$. The sets $\left\{x_{m}, x_{m+1}, x_{m+2}\right\},\left\{x_{2 m}, z, x_{2 m+2}\right\}$ and $\left\{x_{2 m-1}, w, x_{2 m+1}\right\}, m \in \mathbb{Z}$, are the clusters of $\mathscr{A}$. The cluster monomials are monomials in cluster variables belonging to the same cluster. The exchange graph of $\mathscr{A}$ is the brick wall shown here:

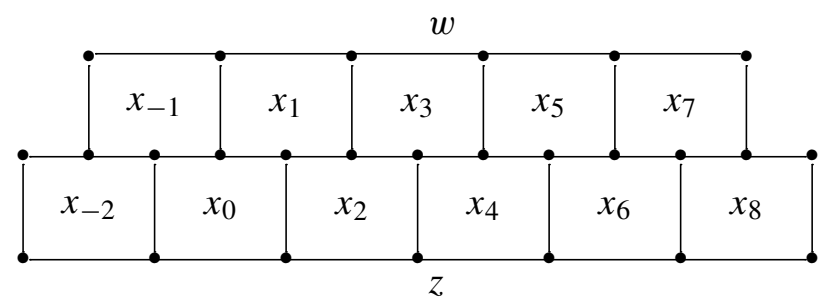

It has clusters as vertices and an edge between two vertices if the corresponding clusters share precisely two cluster variables. In this figure the cluster variables of a cluster $\mathscr{C}$ label the regions surrounding the vertex corresponding to $\mathscr{C}$.

Define elements $u_{n}, n \geq 0$, of $\mathscr{F}$ by the recursion

$$
u_{0}=2, \quad u_{1}=z w-2, \quad u_{n+1}=u_{1} u_{n}-u_{n-1} \quad \text { for } n \geq 1 .
$$

In [Cerulli Irelli 2009] it is shown that the set

$$
\boldsymbol{B}=\{\text { cluster monomials }\} \cup\left\{u_{n} w^{k}, u_{n} z^{k}: n \geq 1, k \geq 0\right\}
$$

is an atomic basis of $\mathscr{A}$. We now realize the elements $u_{n}$ as images of the CalderoChapoton map. Recall that for a representation $M$ of the quiver $Q_{2}$ of (51) the Caldero-Chapoton map $C C(M)$ is the following (see [Caldero and Zelevinsky 2006]):

$$
C C(M)=\frac{\sum_{e} \chi\left(\mathrm{Gr}_{\boldsymbol{e}}(M)\right) x_{1}^{d_{2}+d_{3}-e_{2}-e_{3}} x_{2}^{d_{3}-e_{3}+e_{1}} x_{3}^{e_{1}+e_{2}}}{x_{1}^{d_{1}} x_{2}^{d_{2}} x_{3}^{d_{3}}}
$$


where $\left(d_{1}, d_{2}, d_{3}\right)$ is the dimension vector of $M$. For every $n \geq 1$ let $R_{n, 2}$ be the indecomposable regular $Q_{2}$-representation in an homogeneous tube, i.e.,

$$
R_{n, 2}=\quad k^{n} \stackrel{\nearrow_{J_{n}(0)}}{\longrightarrow} k^{n}
$$

where $J_{n}(0)$ denotes the $n \times n$ indecomposable nilpotent Jordan block.

Theorem 19. For every $n \geq 1$,

$$
u_{n}=\frac{\sum_{\boldsymbol{e}} \chi\left(\mathrm{Gr}_{\boldsymbol{e}}\left(R_{n, 2}\right)^{S m}\right) x_{1}^{2 n-e_{2}-e_{3}} x_{2}^{n-e_{3}+e_{1}} x_{3}^{e_{1}+e_{2}}}{x_{1}^{n} x_{2}^{n} x_{3}^{n}}
$$

where $\mathrm{Gr}_{\boldsymbol{e}}\left(R_{n, 2}\right)^{\text {Sm }}$ denotes the smooth part of the quiver Grassmannian $\mathrm{Gr}_{\boldsymbol{e}}\left(R_{n, 2}\right)$, $\boldsymbol{e}:=\left(e_{1}, e_{2}, e_{3}\right)$.

Proof. Let $u_{n}^{\prime}$ denote the right-hand side of (54). We prove that $\left\{u_{n}^{\prime}\right\}$ satisfies (52). We consider the fibration of projective varieties

$$
f: \operatorname{Gr}_{\left(e_{1}, e_{2}, e_{3}\right)}\left(R_{n, 2}\right) \rightarrow \operatorname{Gr}_{\left(e_{1}, e_{3}\right)}\left(R_{n}\right)
$$

taking $\left(N_{1}, N_{2}, N_{3}\right)$ to $\left(N_{1}, N_{3}\right)$; its fiber is $\operatorname{Gr}_{\left(e_{2}-e_{1}\right)}\left(e_{3}-e_{1}\right)$. In particular

$$
\operatorname{Gr}_{\left(e_{1}, e_{2}, e_{3}\right)}\left(R_{n, 2}\right)^{S m}=f^{-1}\left(\operatorname{Gr}_{\left(e_{1}, e_{3}\right)}\left(R_{n}\right)^{S m}\right) \text {. }
$$

Using this it is straightforward to check that

$$
u_{n}^{\prime}=z_{n}\left(x_{1} w^{-1 / 2}, x_{3} w^{-1 / 2}\right)
$$

where the right-hand side means that (48) should be computed with the substitutions $x_{1} \mapsto x_{1} w^{-1 / 2}$ and $x_{2} \mapsto x_{3} w^{-1 / 2}$, and $w^{-1 / 2}$ is a formal variable whose square is $w^{-1}$. The defining equations (4) yield

$$
u_{1}^{\prime}=z_{1}\left(x_{1} w^{-1 / 2}, x_{3} w^{-1 / 2}\right)=\frac{x_{1}^{2}+x_{3}^{2}+w}{x_{1} x_{2}}=z w-2
$$

and $u_{n+1}^{\prime}=u_{1}^{\prime} u_{n}^{\prime}-u_{n-1}^{\prime}$, so $u_{n}^{\prime}=u_{n}$ for every $n \geq 1$.

\section{Conclusions}

The study of quiver Grassmannians from a geometric point of view has provided interesting information about cluster algebras. After this study it is natural to define the following slight modification of the Caldero-Chapoton map: for $k \geq 0$,

$$
C C^{(k)}(M):=\frac{\sum_{\boldsymbol{e}} \chi\left(\mathrm{Gr}_{\boldsymbol{e}}(M)^{(k)}\right) x_{1}^{2\left(d_{2}-e_{2}\right)} x_{2}^{2 e_{1}}}{x_{1}^{d_{1}} x_{2}^{d_{2}}},
$$


where

$$
\operatorname{Gr}_{\boldsymbol{e}}(M)^{(k)}:=\left\{N \in \operatorname{Gr}_{\boldsymbol{e}}(M): \operatorname{dim} \operatorname{Ext}^{1}(N, M / N)=k\right\} .
$$

In the case of the Kronecker quiver the map $C C^{(0)}(M)$ is defined by the smooth part of the quiver Grassmannians associated with $M$. This is not a general fact and it is studied in [Cerulli Irelli et al. 2012]. In that paper we study the relationship between the maps $C C^{(k)}$ and the transverse quiver Grassmannian introduced in [Dupont 2010].

We notice that for a rigid representation $M$ we have $C C^{(0)}(M)=C C(M)$. Moreover it is easy to check that if $\operatorname{Ext}^{1}(M, N)=\operatorname{Ext}^{1}(N, M)=0$ then $C C^{0}(M \oplus N)=$ $C C^{0}(M) C C^{0}(N)$. In particular the elements $u_{n} w$ and $u_{n} z$ of the basis (53) are $C C^{0}\left(R_{n, 2} \oplus W\right)$ and $C C^{0}\left(R_{n, 2} \oplus Z\right)$ where $W$ and $Z$ are the indecomposable regular rigid $Q_{2}$-representations. We trust that this approach can help in the determination of atomic bases of cluster algebras of affine type of higher rank.

\section{Acknowledgements}

We thank Andrea Maffei for useful conversations.

Cerulli Irelli thanks the organizers of the "Advanced School and Conference on Homological and Geometrical Methods in Representation Theory" that took place in Trieste (Italy) at the beginning of 2010 for the opportunity to present this project in front of several experts and to spend a fruitful time there.

We are grateful to Andrei Zelevinsky for having pointed out the formulas in [Szanto 2011], and to Grégoire Dupont for his comments about transverse quiver Grassmannians.

\section{References}

[Assem et al. 2006] I. Assem, D. Simson, and A. Skowroński, Elements of the representation theory of associative algebras, vol. 1: Techniques of representation theory, London Math. Soc. Student Texts 65, Cambridge University Press, Cambridge, 2006. MR 2006j:16020 Zbl 1092.16001

[Berenstein et al. 2005] A. Berenstein, S. Fomin, and A. Zelevinsky, "Cluster algebras, III: Upper bounds and double Bruhat cells”, Duke Math. J. 126:1 (2005), 1-52. MR 2005i:16065

[Białynicki-Birula 1973] A. Białynicki-Birula, "Some theorems on actions of algebraic groups", Ann. of Math. (2) 98 (1973), 480-497. MR 51 \#3186 Zbl 0275.14007

[Caldero and Chapoton 2006] P. Caldero and F. Chapoton, "Cluster algebras as Hall algebras of quiver representations”, Comment. Math. Helv. 81:3 (2006), 595-616. MR 2008b:16015 Zbl 1119. 16013

[Caldero and Keller 2006] P. Caldero and B. Keller, "From triangulated categories to cluster algebras, II”, Ann. Sci. École Norm. Sup. (4) 39:6 (2006), 983-1009. MR 2008m:16031 Zbl 1115.18301

[Caldero and Keller 2008] P. Caldero and B. Keller, "From triangulated categories to cluster algebras", Invent. Math. 172:1 (2008), 169-211. MR 2009f:16027 Zbl 1141.18012

[Caldero and Reineke 2008] P. Caldero and M. Reineke, "On the quiver Grassmannian in the acyclic case”, J. Pure Appl. Algebra 212:11 (2008), 2369-2380. MR 2009f:14102 Zbl 1153.14032 
[Caldero and Zelevinsky 2006] P. Caldero and A. Zelevinsky, "Laurent expansions in cluster algebras via quiver representations", Mosc. Math. J. 6:3 (2006), 411-429. MR 2008j:16045 Zbl 1133.16012

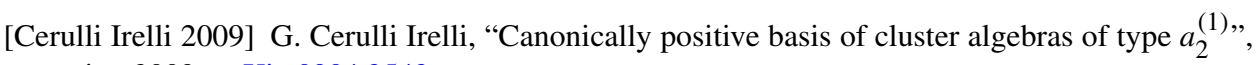
preprint, 2009. arXiv 0904.2543

[Cerulli Irelli 2011a] G. Cerulli Irelli, "Positivity in skew-symmetric cluster algebras of finite type", preprint, 2011. arXiv 1102.3050

[Cerulli Irelli 2011b] G. Cerulli Irelli, "Quiver grassmannians associated with string modules", $J$. Algebraic Combin. 33:2 (2011), 259-276. MR 2765325 Zbl 05852822

[Cerulli Irelli and Labardini-Fragoso 2011] G. Cerulli Irelli and D. Labardini-Fragoso, "Quivers with potentials associated to triangulated surfaces, III: tagged triangulations and cluster monomials", (2011). arXiv 1108.1774

[Cerulli Irelli et al. 2012] G. Cerulli Irelli, G. Dupont, and F. Esposito, "A homological interpretation of the transverse quiver grassmannians", Algebr. Represent. Theory (2012).

[Chriss and Ginzburg 1997] N. Chriss and V. Ginzburg, Representation theory and complex geometry, Birkhäuser, Boston, 1997. MR 98i:22021 Zbl 0879.22001

[Crawley-Boevey 1989] W. W. Crawley-Boevey, "Maps between representations of zero-relation algebras", J. Algebra 126:2 (1989), 259-263. MR 90k:16035 Zbl 0685.16018

[Derksen et al. 2010] H. Derksen, J. Weyman, and A. Zelevinsky, "Quivers with potentials and their representations, II: Applications to cluster algebras”, J. Amer. Math. Soc. 23:3 (2010), 749-790. MR 2629987

[Dupont 2010] G. Dupont, "Transverse quiver grassmannians and bases in affine cluster algebras", Algebra Number Theory 4:5 (2010), 599-624. MR 2011i:13027 Zbl 05779836

[Fomin and Zelevinsky 2002] S. Fomin and A. Zelevinsky, "Cluster algebras, I: Foundations", J. Amer. Math. Soc. 15:2 (2002), 497-529. MR 2003f:16050

[Fomin and Zelevinsky 2003a] S. Fomin and A. Zelevinsky, "Cluster algebras. II. Finite type classification”, Invent. Math. 154:1 (2003), 63-121. MR 2004m:17011

[Fomin and Zelevinsky 2003b] S. Fomin and A. Zelevinsky, "Cluster algebras: notes for the CDM-03 conference", pp. 1-34 in Current developments in mathematics, 2003, edited by B. Mazur et al., Int. Press, Somerville, MA, 2003. MR 2005m:05235 Zbl 1119.05108

[Fomin and Zelevinsky 2007] S. Fomin and A. Zelevinsky, "Cluster algebras, IV: Coefficients", Compos. Math. 143:1 (2007), 112-164. MR 2008d:16049

[Geiß et al. 2012] C. Geiß, B. Leclerc, and J. Schröer, "Generic bases for cluster algebras and the chamber ansatz", J. Amer. Math. Soc. 25:1 (2012), 21-76. MR 2833478

[Hernandez and Leclerc 2010] D. Hernandez and B. Leclerc, "Cluster algebras and quantum affine algebras”, Duke Math. J. 154:2 (2010), 265-341. MR 2011g:17027 Zbl 05788166

[Keller 2010] B. Keller, "Cluster algebras, quiver representations and triangulated categories", pp. 76-160 in Triangulated categories, edited by T. Holm et al., London Math. Soc. Lecture Note Ser. 375, Cambridge Univ. Press, Cambridge, 2010. MR 2011h:13033 Zbl 1215.16012

[Kronecker 1890] L. Kronecker, "Algebraische Reduction der Schaaren bilinearer Formen", Sitzungsber. Akad. Berlin (1890), 1225-1237. Reprinted as pp. 139-156 in his Werke, vol. 3, Teubner, Leipzig, 1931. JFM 22.0169.01

[Lampe 2011] P. Lampe, "A quantum cluster algebra of Kronecker type and the dual canonical basis", Int. Math. Res. Not. 2011:13 (2011), 2970-3005. MR 2817684 Zbl 05931742

[Nakajima 2011] H. Nakajima, "Quiver varieties and cluster algebras”, Kyoto J. Math. 51:1 (2011), 71-126. MR 2784748 Zbl 1223.13013 
[Poettering 2010] N. Poettering, "Euler characteristic of quiver Grassmannians and Ringel-Hall algebras of string algebras", preprint, 2010. To appear in Algebr. Represent. Theory. arXiv 1002.3040

[Ringel 1998] C. M. Ringel, "Exceptional modules are tree modules", Linear Algebra Appl. 275/276 (1998), 471-493. MR 2000c:16020 Zbl 0964.16014

[Sherman and Zelevinsky 2004] P. Sherman and A. Zelevinsky, "Positivity and canonical bases in rank 2 cluster algebras of finite and affine types", Mosc. Math. J. 4:4 (2004), 947-974. MR 2006c: 16052 Zbl 1103.16018

[Szanto 2011] C. Szanto, "On the cardinalities of Kronecker quiver grassmannians", Math. Z. 269:3-4 (2011), 833-846.

[Zelevinsky 2007] A. Zelevinsky, "Semicanonical basis generators of the cluster algebra of type

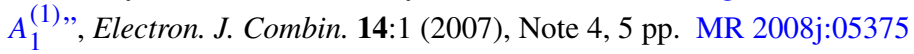

Communicated by Andrei Zelevinsky

Received 2010-03-22 Revised 2010-09-14 Accepted 2010-10-30

cerulli@mat.uniroma1.it

Current address:

esposito@math.unipd.it
Università degli studi di Padova, Dipartimento di Matematica Pura ed Applicata, Via Trieste 63, I-35121 Padova, Italy

Università di Roma "La Sapienza", Dipartimento di Matematica, Piazzale Aldo Moro 5, I-00185 Roma, Italy

Università degli studi di Padova, Dipartimento di Matematica Pura ed Applicata, Via Trieste 63, I-35121 Padova, Italy 


\section{Algebra \& Number Theory}

msp.berkeley.edu/ant

\section{EDITORS}

MANAGING EDITOR

Bjorn Poonen

Massachusetts Institute of Technology

Cambridge, USA

\author{
EDITORIAL BOARD CHAIR \\ David Eisenbud \\ University of California \\ Berkeley, USA
}

\section{BOARD OF EDITORS}

Georgia Benkart

Dave Benson

Richard E. Borcherds

John H. Coates

J-L. Colliot-Thélène

Brian D. Conrad

Hélène Esnault

Hubert Flenner

Edward Frenkel

Andrew Granville

Joseph Gubeladze

Ehud Hrushovski

Craig Huneke

Mikhail Kapranov

Yujiro Kawamata

János Kollár

Yuri Manin

Barry Mazur

Philippe Michel

Susan Montgomery
University of Wisconsin, Madison, USA

University of Aberdeen, Scotland

University of California, Berkeley, USA

University of Cambridge, UK

CNRS, Université Paris-Sud, France

University of Michigan, USA

Universität Duisburg-Essen, Germany

Ruhr-Universität, Germany

University of California, Berkeley, USA

Université de Montréal, Canada

San Francisco State University, USA

Hebrew University, Israel

University of Kansas, USA

Yale University, USA

University of Tokyo, Japan

Princeton University, USA

Northwestern University, USA

Harvard University, USA

École Polytechnique Fédérale de Lausanne

University of Southern California, USA
Shigefumi Mori

Raman Parimala

Jonathan Pila

Victor Reiner

Karl Rubin

Peter Sarnak

Joseph H. Silverman

Michael Singer

Ronald Solomon

Vasudevan Srinivas

J. Toby Stafford

Bernd Sturmfels

Richard Taylor

Ravi Vakil

Michel van den Bergh

Marie-France Vignéras

Kei-Ichi Watanabe

Andrei Zelevinsky

Efim Zelmanov
RIMS, Kyoto University, Japan

Emory University, USA

University of Oxford, UK

University of Minnesota, USA

University of California, Irvine, USA

Princeton University, USA

Brown University, USA

North Carolina State University, USA

Ohio State University, USA

Tata Inst. of Fund. Research, India

University of Michigan, USA

University of California, Berkeley, USA

Harvard University, USA

Stanford University, USA

Hasselt University, Belgium

Université Paris VII, France

Nihon University, Japan

Northeastern University, USA

University of California, San Diego, USA

\section{PRODUCTION}

contact@msp.org

Silvio Levy, Scientific Editor

See inside back cover or www.jant.org for submission instructions.

The subscription price for 2011 is US \$150/year for the electronic version, and \$210/year (+\$35 shipping outside the US) for print and electronic. Subscriptions, requests for back issues from the last three years and changes of subscribers address should be sent to Mathematical Sciences Publishers, Department of Mathematics, University of California, Berkeley, CA 94720-3840, USA.

Algebra \& Number Theory (ISSN 1937-0652) at Mathematical Sciences Publishers, Department of Mathematics, University of California, Berkeley, CA 94720-3840 is published continuously online. Periodical rate postage paid at Berkeley, CA 94704, and additional mailing offices.

ANT peer review and production are managed by EditFLOW ${ }^{\circledR}$ from Mathematical Sciences Publishers.

PUBLISHED BY

mathematical sciences publishers

http://msp.org/

A NON-PROFIT CORPORATION

Typeset in IAT $_{\mathrm{E}} \mathrm{X}$

Copyright @2011 by Mathematical Sciences Publishers 


\section{Algebra \& Number Theory}

Volume $5 \quad$ No. $6 \quad 2011$

Higher direct images of the structure sheaf in positive characteristic

ANDRE CHATZISTAMATIOU and KAY RÜLLING

Geometry of quiver Grassmannians of Kronecker type and applications to cluster algebras

Giovanni Cerulli Irelli and Francesco Esposito

Sur le groupe de Chow de codimension deux des variétés sur les corps finis

ALENA PIRUTKA

Sur les invariants d'Iwasawa dans les extensions de Lie $p$-adiques

Guillaume Perbet 